\title{
DES CATÉGORIES SPATIALES À L'ALTÉRITÉ TERRITORIALE : POUR UNE MEILLEURE CONSIDÉRATION DES SINGULARITÉS ET DES PRATIQUES SOCIO-SPATIALES DANS L'ACTION LOCALE
}

\author{
Mélanie GAMBINO, Mariette SIBERTIN-BLANC
}

\begin{abstract}
Résumé
À partir d'une recherche qualitative menée en Région Occitanie interrogeant les catégories spatiales (urbaines, périurbaines, rurales), l'article met en lumière l'importance dans les discours des acteurs interrogés de l'altérité territoriale, soit une conscience des différenciations territoriales et le besoin formulé d'identifier les territoires de vie vis-à-vis d'autres territoires. Le discours des acteurs opérationnels et des habitants interrogés souligne à la fois les ressorts de l'altérité territoriale (trajectoires de vie, expérience, etc.), mais aussi les capacités requises pour maitriser des agencements et les interactions de la vie quotidienne : capacités de mobilité, d'adaptation, d'intégration. Ces différentes capacités contribuent à un capital d'autochtonie susceptible d'être multi-situé, ce qui interroge les objectifs et dispositifs de développement territorial. Aussi faisons-nous l'hypothèse que l'analyse des représentations et des pratiques socio-spatiales, peu considérée dans les travaux de recherche sur cette problématique, est susceptible d'éclairer à la fois de nouvelles relations à l'espace, mais aussi de nouveaux enjeux pour le pilotage de l'action territoriale.
\end{abstract}

\begin{abstract}
Mots-clés
urbain, rural, développement territorial, modes d'habiter, différentiation territoriale, analyse qualitative de discours

Summary

Based on a qualitative survey carried out in Occitanie Region (France) questioning spatial categories (urban, peri-urban, rural), the paper highlights the importance in discourse of actors interviewed of territorial otherness, that is to say an awareness of territorial differentiation and the need to identify living territories vis-à-vis other territories. Discourses of operational actors and inhabitants highlights both drivers of territorial otherness (life trajectories, experience, etc.), and capacities required to master social arrangements and interactions of everyday life: mobility, adaptation and integration capacities. These different capacities contribute to a local spatial capital likely to be multi-situated, which questions territorial development's objectives and schemes. We therefore hypothesize that the analysis of socio-spatial representations and practices, which is little considered in academic research on that matter, is likely to shed light on both new relationships to place and new issues to manage territorial action.
\end{abstract}

\author{
Keywords \\ urban, rural, territorial development, livelihoods, territorial distinction, qualitative discourse \\ analysis
}

\section{INTRODUCTION}

La question de l'(in)adéquation entre territoires vécus et territoires administrés n'est pas nouvelle (Ascher, 1995 ; Arlaud et al., 2005), tout comme celle de la cohérence des catégories et oppositions spatiales pour décrire les réalités locales (Béhar, 2018). Bien que ces dernières années aient été favorables à la mise en lumière des différenciations territoriales, à l'instar d'un projet de loi en France intégrant directement le terme (Jebeili, 2019), force est de constater qu'il est encore difficile de rencontrer dans les politiques publiques la fine intégration de la complexité des pratiques socio-spatiales (Di
Méo, 1996). Sans poursuivre l'ambition d'une recette empirique magique, il s'agit ici, à partir d'un corpus d'entretiens (acteurs opérationnels et habitants, cf encadré méthodologique), d'illustrer la grande diversité des représentations territoriales et l'importance, pour tous, d'insister sur la distinction que nous définirons comme l'altérité territoriale soit le besoin de positionnement de son lieu de vie en le comparant à d'autres selon une singularité socio-spatiale.

Si les dynamiques territoriales de l'espace français ne sont plus aujourd'hui structurées par une dualité urbain-rural (Lévy, 2013 ; Charmes, 2017) mais par 
un ensemble de fonctions, de systèmes d'organisation de l'espace révélant des logiques d'imbrication entre différentes réalités socio-spatiales et des mutations du système productif (Talandier et Pecqueur, 2018), d'aucuns considèrent les espaces sous une influence généralisée de l'urbain (comme l'atteste le titre de revue Tous urbains), d'autres tentent de considérer non seulement les formes de villes qui se dessinent sous le processus de métropolisation (de la métapole de F. Ascher en 1995 à l'archipel métropolitain de N. Cattan en 2017), mais aussi les nouvelles logiques à l'œuvre en termes de territorialisation des pratiques (sociales, politiques, économiques).

Dans cette perspective, les catégories et oppositions spatiales du rural et de l'urbain, encore à l'œuvre dans les mondes académique et médiatique, sont-elles encore pertinentes pour expliquer les dynamiques territoriales, et donc pour appuyer l'action publique et être support de dispositifs et d'actions d'un développement contextualisé et territorialisé ? Nous faisons l'hypothèse que l'analyse des représentations et des pratiques socio-spatiales, peu considérées dans les travaux de recherche sur cette problématique, est susceptible d'éclairer à la fois de nouvelles relations à l'espace, des capacités individuelles à intégrer dans les objectifs de développement territorial, et donc de nouveaux enjeux pour le pilotage de l'action territoriale. Il s'agit ici de considérer une complexité territoriale issue du rapport sensible et individualisé à l'espace, des pratiques socio-spatiales, qui expriment des besoins particuliers dont la connaissance et la prise en compte est importante pour orienter les politiques publiques. Notre analyse sur les catégories et les processus de différenciations opérés par les habitants s'ajoute aux travaux portant sur les territoires ordinaires et leur place dans les politiques territoriales (Vanier, 2010 ; Cailly et Pourteau, 2018 ; Offner, 2019).

Aussi, après avoir rappelé la diversité de représentations spatiales qui insiste sur l'altérité territoriale, l'exploitation de l'enquête qualitative auprès d'habitants de territoires très différenciés rendra compte de la complexification du rapport à l'espace, mettant en exergue certaines capacités pour bien vivre les contrastes spatiaux construits, et l'importance d'un capital souvent minoré : celui d'autochtonie.
Encadré 1 : Méthode et terrains d'étude

L'analyse s'appuie sur une méthode qualitative développée dans le programme Interactions, du Labex Structuration des Mondes Sociaux; les matériaux analysés proviennent d'entretiens semi-directifs avec d'une part des habitants et d'autre part des acteurs opérationnels de terrain (élus, techniciens notamment en planification et développement territorial).

9 terrains d'un transect nord-sud de l'ouest de l'Occitanie (ex Midi-Pyrénées) ont été investigués à l'échelle intercommunale (Figure 1), croisant divers profils spatiaux selon les catégories spatiales produites par l'Institut national de la statistique et des études économiques (INSEE) et le Commissariat général à l'égalité des territoires (CGET), avec la variété des périmètres (Parc Naturel Régional (PNR), Pôle d'équilibre territorial et rural (Petr), Communautés d'agglomération et de communes, Métropole). La diversité des territoires retenus permet de rencontrer des configurations objectives variées, mais aussi différents périmètres politiques de conduite de projet, et formes d'interactions rural-urbain.

L'enquête sur les pratiques socio-spatiales (Di Méo, 1996) repose sur 54 entretiens réalisés en 2015 , auprès d'habitants entre 29 et 50 ans et davantage de femmes (30) que d'hommes (24), dont le lieu de résidence relevait d'une diversité de catégorie spatiale statistique. Volontairement, l'échantillon n'a pas privilégié un profil socio-professionnel dominant : 5 enquêtés sont agriculteurs, 8 artisans, commerçants et chefs d'entreprises, 6 cadres et professions intellectuelles supérieures, 10 professions intermédiaires, 15 employés, 2 ouvriers, 3 sans activité professionnelle et 6 non communiquées. Chaque enquêté a eu la possibilité de faire un schéma illustrant son territoire vécu (ex. Figure 2). L'objectif de ces entretiens était prioritairement de comprendre comment les catégories spatiales sont mobilisées dans les modes d'habiter, et quelles sont les interactions entre les différents types de territoires et dans quelle mesure les trajectoires de vie et les mobilités construisent des représentations de la différenciation territoriale. Le guide d'entretien auprès des habitants portait sur les trajectoires 

singularités et des pratiques socio-spatiales dans l'action locale

résidentielles, les qualifications des lieux de vie entre urbain et rural, les mobilités et le rapport à la nature de ces habitants et a été appliqué également quel que soit le profil territorial enquêté. Il proposait également une représentation graphique des territoires vécus (Figure 2).

En parallèle des entretiens avec les habitants, 15 entretiens portant sur l'évolution récente des périmètres, le développement d'un projet de territoire et les relations avec d'autres territoires (voisins ou supra) ont été réalisés dans chaque EPCI avec un ou deux élus (le président ou un délégué à l'urbanisme et à l'aménagement du territoire), un directeur de services (d'urbanisme ou le directeur général des services) ainsi que des chargés de mission dans les structures porteuses de SCOT, PNR et PETR. Il s'agissait dans ces entretiens de considérer également dans quelle mesure les catégories spatiales étaient mobilisées pour l'action et les stratégies locales. Comme pour les habitants, la question des différences territoriales était abordée.

\section{RURAL, URBAIN, MÉTROPOLE, FAIBLE DENSITÉ, ... : UNE MULTITUDE DE REPRÉSENTATIONS DU MONDE}

Au regard des catégories statistiques, l'opposition ville-campagne ou les différences rural-urbain sont toujours mobilisées pour tenter d'éclairer les enjeux et fonctionnements locaux. Si des typologies sont proposées par différents opérateurs (INSEE, DATAR puis CGET) pour nuancer les positions tranchées qui consistent à considérer que "rien n'échappe à l'urbain » (Lussault, 2018), elles semblent toutefois peu mobilisées pour construire l'action publique locale. C'est ainsi qu'à la suite d'un travail approfondi sur l'analyse de ces différentes typologies spatiales (Barthe et al., 2017), nous avions conclu à ce que la complexité du croisement des indicateurs et le fait qu'aucune typologie ne se recoupe traduisent la complexité de l'interprétation des dynamiques territoriales mais interrogent aussi sur la façon dont les acteurs locaux peuvent s'en saisir. Ces différentes façons de rendre compte des réalités spatiales donnent l'occasion aux acteurs territoriaux de s'inscrire « banalement» dans des catégories les plus communes, particulièrement celles de l'INSEE, ou au contraire d'en faire abstraction et de mobiliser leurs propres représentations fondées sur le vécu quotidien des acteurs et habitants.

\section{A. Représentations politiques et opérationnelles : focus sur les contrastes territoriaux et les enjeux de développement}

Les résultats des enquêtes menées auprès des acteurs du champ opérationnel dans le transect où se situent les neuf terrains étudiés montrent que si les dynamiques territoriales sont marquées par les interactions et le fonctionnement des dispositifs par l'interterritorialité (Vanier, 2008), la lecture politique de ces dynamiques se fait encore sur le mode de la dualité et de l'opposition : entre ville et campagne, entre catégories de l'urbain et du rural, entre le centre et la France périphérique, entre les métropoles et les hinterlands. Le besoin de se situer dans l'espace et de s'identifier par rapport à d'autres constitue une posture partagée, avec des nuances mais aussi, comme le montrent les résultats de l'enquête auprès des acteurs opérationnels, des tendances lourdes. Les documents de planification et les discours des acteurs interrogés dans les différents territoires laissent entrevoir une représentation territoriale contrastée, voire parfois caricaturale, avec souvent une perspective stratégique. Les acteurs locaux énoncent une représentation de leur territoire, en tant qu'espace investi par des représentations et des modes d'actions et d'organisation, s'appuyant sur un modèle dominant centre-périphérie. Alors que la région Midi-Pyrénées est historiquement structurée par une métropole unique, Toulouse, et par des villes petites et moyennes dont les caractéristiques reflètent la dominante départementale (bourgs agricoles, petites villes industrielles, pôles administratifs), l'importance de la hiérarchie urbaine est souvent rappelée, s'appuyant sur la structuration des flux (en particulier liés aux chaînes de production telle que celle de l'aéronautique), la réalité des mobilités domicile-travail mais aussi l'accessibilité des services publics :

"Ici c'est du rural, à l'exception de deux villages et de Millau qui sont dotés d'infrastructures publiques : maisons médicales, écoles, etc. » (directeur de Cabinet du Maire de Millau, parlant du territoire du millavois).

Cette citation illustre une approche par distinction fonctionnelle des espaces, qui se retrouve dans un clivage plus global : la ville considérée comme 


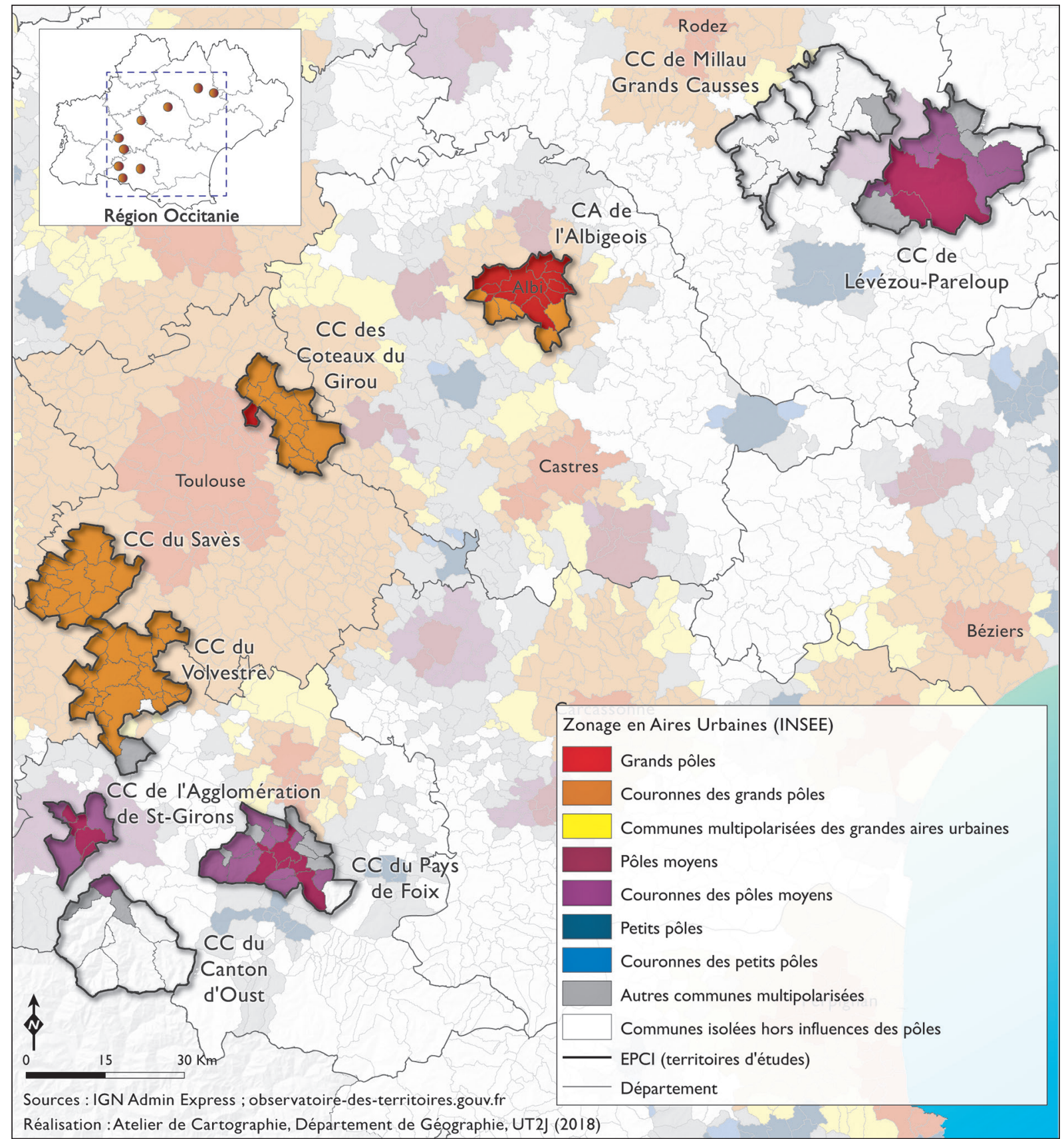

Figure 1. Localisation des 9 terrains d'étude

un espace commercial et de services, caractérisée par son attractivité voire son rôle asséchant, et la campagne, comme un espace agricole et, peut-être paradoxalement, où la nature est préservée :

"Toulouse, c'est la métropole qui absorbe toute la richesse et l'emploi » (Entretien avec le Maire de Foix)

"L'espace environnemental et naturel fait ressource » (Directrice de la Communauté de commune de Lévezou Pareloup)

"Ici c'est rural, les tracteurs et les bœufs se baladent dans le village » (Directeur du service urbanisme de Saint-Girons)
Ainsi, les contrastes insistent-ils sur les jeux d'oppositions, renforcés par une approche sensible, mettant en avant le cadre de vie et la qualité paysagère des espaces. C'est ce que révèle une élue d'une commune pourtant très touchée par l'étalement urbain :

«Contrairement aux banlieues toulousaines ravagées, nos paysages sont extrêmement préservés" (Adjointe à l'urbanisme de la CA de l'Albigeois, Maire de Marssac sur Tarn)

«Notre atout c'est le cadre de vie, c'est d'avoir un territoire agréable, on y est quand même mieux [qu'à Toulouse] » (Élue communautaire de la Communauté de Commune du Volvestre) 

singularités et des pratiques socio-spatiales dans l'action locale

En analysant les verbatim des acteurs locaux, leurs représentations spatiales et les problématiques rencontrées par les territoires, il apparaît clairement que le récit formulé sur «sa » catégorie reflète des préoccupations de développement territorial spécifiques : le déséquilibre interne dans la hiérarchie urbaine et la place de la ville d'Albi au sein d'un SCOT (Schéma de cohérence territoriale) ; la mutation de l'identité agricole dans le SCOT Sud Toulousain qui enregistre une transformation sociale et d'artificialisation des sols très rapides ; les caractéristiques montagnardes de certains territoires, pour lesquels la gestion des espaces naturels et des conflits d'usage prédomine. Il apparaît ainsi que la représentation des catégories spatiales et les caractéristiques de différenciation sont associées aux trajectoires de développement qu'il s'agit d'accompagner (mise en tourisme, gestion de la crise industrielle, accueil de nouveaux profils de population, étalement urbain, etc.).

Comme pour les représentations des habitants présentés ci-après, les acteurs produisent des discours sur leur territoire qui s'appuient sur la volonté d'identification. Celle-ci est focalisée sur le positionnement dans le système urbain régional pour lequel l'organisation mono-centrique de la région ex-Midi-Pyrénées est déterminante. Une altérité territoriale est donc revendiquée, positionnant le territoire vis-à-vis du pôle métropolitain de Toulouse. Trois modèles sont identifiables. Le premier, défensif, concerne les territoires qui se revendiquent plutôt comme étant territoire rural, défendant un cadre de vie où la nature est présente et constitue un atout. Malgré les flux, voire les dépendances vis-à-vis de la métropole (emplois et salaires, services et équipements), les acteurs de ces territoires considèrent qu'ils ont à se protéger d'un type de développement territorial qu'ils souhaitent mettre à distance. Le deuxième, offensif, a pris acte du processus de métropolisation et les acteurs relaient une stratégie de développement qui tente d'en tirer parti. Les villes moyennes intégrées dans le dialogue métropolitain sont représentatives de ce modèle (Albi, Foix), ainsi que certains PETR, dont les intérêts sont avant tout considérés du côté de la réciprocité. Enfin, un dernier modèle se dégage, celui de la distinction, autour des territoires qui cherchent à ignorer des processus métropolitains relativement lointains. Se considérant comme coupés des logiques des grandes villes, les territoires de faible densité (Couserans, Lévezou) cherchent à construire des projets de territoire fondés sur l'activation de leurs ressources spécifiques (montagnardes, frontalières).

Basés essentiellement sur le positionnement vis-àvis d'autres territoires, ces discours opérationnels mettent en avant certains aspects qui justifient les politiques publiques locales mises en place, sans toutefois revendiquer des logiques statistiques ou de zonage (aire urbaine, périurbain, rural isolé, etc.). Alors que les représentations des acteurs territoriaux interrogés s'affranchissent de la composante sociale et de la diversité des modes d'habiter des territoires, les habitants construisent pourtant, également, des représentations construites sur l'altérité territoriale.

\section{B. La parole habitante : diversification des regards, expériences territoriales et émotionnelles}

Un nouveau regard sur les réalités territoriales est apporté par les discours des habitants interrogés sur leurs pratiques et leurs représentations de l'espace. Le choix méthodologique qui a prévalu à ces résultats permet d'aborder à la fois la question des catégories spatiales telles que vécues par les habitants, mais aussi leurs interactions révélées dans des pratiques quotidiennes ou plus distendues. Aussi le passage de l'espace au territoire selon Di Méo (1991) prend-il tout son sens : «Sur le socle que dresse la réalité socioculturelle, le territoire témoigne d'une appropriation à la fois économique, idéologique et politique (sociale donc) de l'espace par des groupes qui se donnent une représentation particulière d'euxmêmes, de leur histoire, de leur singularité ».

En premier lieu, si les distinctions spatiales existent, les catégories « rural-urbain » sont quasi absentes des discours, tout comme les nouveaux périmètres (EPCI, PETR, etc.) qui sont rarement connus par les personnes interrogées. Par contre, des termes utilisés illustrent une identification plus fine, renvoyant d'une part à la hiérarchie urbaine («petite ville », « grande ville »), infra urbain (« quartier », «banlieue»), et d'autre part à des termes relatifs au cadre paysager : « la campagne », « la montagne». Un grand absent peut être pointé dans les discours : le périurbain n'existe pas dans les représentations spatiales spontanées, comme observé en 2007 (Morel-Brochet) même si des pratiques telles que les achats en grandes surfaces périphériques peuvent être évoquées, mais associées à la " sortie en ville» de ceux qui sont aux extrémités du transect étudié. 
De manière plus transversale, la notion d'altérité territoriale peut être remobilisée et déclinée ci-dessous, car les habitants interrogés, quels qu'ils soient, construisent de la différenciation qui s'appuie sur quatre dimensions.

\section{Des représentations " sensibles 》 et expérien- tielles du territoire}

Elles sont liées aux trajectoires résidentielles et à la qualité affective et sensorielle de ce qui a été vécu. Par exemple, c'est le cas pour Blandine (qui vit aujourd'hui à Saint-Girons, 43 ans) : selon elle, Barcelone, où une belle histoire d'amour a été vécue, est « la bonne échelle de ville», permettant « des relations humaines conviviales et chaleureuses 》... alors que Paris, où elle a vécu un temps de «galère professionnelle », est considérée comme une strate urbaine où règne « agressivité, fausseté, anonymat». Pour ce qui est de la petite ville où des perspectives personnelles semblent manquer, elle devient « enfermante ». Ainsi l'utilisation des adjectifs renvoient à la qualité de ce qui a été traversé pour décrire le territoire dans lequel ces expériences ont été vécues.

Cette approche associant profil spatial et appréciation qualitative de ce qui est vécu se retrouve dans d'autres cas :

«En ville, c'est l'agitation ambiante et constante. Juste la fatigue de... Même quand on arrête de parler, il y a toujours quelque chose qui se passe, en fait. Et ça, c'est épuisant. " (Magali, Rieumes, 30 ans, animatrice environnement).

«St Girons, la petite ville où on a tout, mais donc on s'y referme. » ou «C'est une petite ville c'est pas du tout agressif, on connaît les gens, c'est même enveloppant... » (Patricia, Saint Girons, 34 ans, Chargée de mission dans une communauté de commune)

2. Une place de la nature qui se retrouve dans tous les discours, quel que soit le profil des territoires

Le rapport à la nature contribue à caractériser les espaces, et à les distinguer, soit en tant que décors, soit en tant que support de pratiques récréatives : promenade, pêche. Dans tous les cas, il s'avère que l'intensité d'urbanisation du lieu de résidence n'est pas corolaire à la place accordée à la nature et à sa qualité.

"Ici, on respire, l'air est bon! La montagne, le vent, les oiseaux. On profite de la nature, on voit le chan- gement avec les saisons » (Blandine, Saint Girons, 43 ans, artiste).

"Mais bon, j'étais quand même entourée de maisons, de routes, de feux rouges, de... Et ici, je regarde dehors, je vois de la verdure... Et c'est ça, pour moi, la nature. Pouvoir tourner mon regard dans tous les sens et avoir l'impression d'être dehors » (Anne, Salles-Curan, 35 ans, institutrice) "Si je peux trouver un chemin, je suis plus heureux, quoi. Mais voilà. Après... Je suis conscient qu'il peut pas y en avoir partout et tout le temps à disposition, mais je veux dire par là : aujourd'hui, on peut en trouver, ici. » (Pierre, Millau, 46 ans, commercial)

Contrairement à une hypothèse envisagée initialement, la nature et sa considération (en tant que décors ou support récréatif) ne peuvent être corrélées à une catégorie, à un niveau de densité, ou à une valeur intrinsèque. La nature, même artificielle, est mobilisée pour qualifier l'urbain.

Si les pratiques décrites sont variées, l'une est soulignée par 29 des 51 interviewés : le rapport à la nature s'apprend et se transmet (souvent dans un cadre familial); il est donc socialisé même s'il est souvent décrit comme une expérience personnelle à l'écart de la société.

"Il y a de la nature en ville, aussi. Et bon, ça, c'est grâce à mes études que j'en ai pris conscience! » (Magali, Rieumes, 30 ans, animatrice environnement)

3. Des contrastes et des oppositions entre profils de territoires

Les habitants mobilisent des différenciations territoriales pour expliquer le monde et justifier leurs modes d'habiter. Un thème global structurant le corpus a été identifié : une opposition d'une part, de traits attribués aux plus grandes villes, comme le bruit, l'agressivité, le trafic routier et la pollution, mais aussi l'accès facilité à l'emploi et aux services, et, d'autre part, la nature qualifiée de sauvage, reliée à l'isolement de la société et l'éloignement des services. Suivant les entretiens, ce sont différents pôles de cette opposition qui sont éclairés. Le rapport au temps est également très souvent mobilisé pour créer ces contrastes.

"On n'est pas stressés ici parce que je sais qu'en ville les enfants ils entendent 20 fois dans la jour- 

singularités et des pratiques socio-spatiales dans l'action locale

née : dépêche-toi on va être en retard... » (Sarah, Saint Girons, 41 ans, adjointe au CCAS).

Ce besoin d'opposition peut se faire par ailleurs à partir d'aspects relativement inattendus, à l'instar de la déclaration d'une habitante d'un village pyrénéen de moins de 1000 habitants :

«Marseille c'est une grosse ville, c'est des immeubles, c'est du béton. Ici, on peut aller à pied n'importe où alors qu'à Marseille on doit prendre la voiture si on veut un peu balader en campagne, pour sortir de la ville!»

\section{Une hiérarchisation et des interactions liées à des pratiques de mobilité (résidentielle, annuelle, quotidienne)}

On repère dans les discours des enquêtés une recherche de validation de leurs propres représentations de la campagne et de la ville. Du côté des habitants des espaces de faible densité, seuls sont évoqués ou détaillés les pratiques et déplacements qui permettent de vérifier au quotidien que la campagne est synonyme d'agricole, de calme, l'opposant ainsi à la ville qui se caractérise chez les enquêtés par les embouteillages et le temps perdu. Cette différenciation accentuée du rural et de l'urbain se retrouve chez Camille (52 ans), commerçante à Foix qui fait les trajets Montgaillard-Foix tous les jours pour le travail, sauf le lundi son seul jour de repos. La mobilité de Camille est essentiellement professionnelle et semble subie, vécue comme une contrainte et inscrite dans la routine quotidienne. Cette mobilité professionnelle semble traduire un héritage de pratiques et la reproduction familiale d'un modèle (sa mère travaillant et se déplaçant avec elle). Le lundi elle en profite pour se reposer, faire « tout ce qu'elle n'a pas pu faire la semaine » (courses, médecin...). Les mobilités consacrées aux loisirs et aux courses d'habillement ou d'ameublement se font à Foix ou dans un grand centre commercial aux portes de Toulouse. Peu nombreuses et secondaires dans la vie de Camille, ces mobilités dites « choisies » s'ajoutent à quelques mobilités touristique ou vacancière présentes mais de moins en moins nombreuses (elle se rend 1 à 2 fois par an à la mer à St Cyprien, sa mère y possédant un appartement). Cette forme de mobilité semble de plus en plus révolue aujourd'hui et fait appel à de lointains souvenirs, ceux du départ en vacances avec ses parents en Espagne durant 1 mois, lorsqu'elle avait 18 ans.
Le besoin de revendiquer une certaine différence dans les pratiques, dans les rapports au territoire et l'identification discursive des différences spatiales (liées à la nature, à la densité, à l'intensité des services ou de l'emploi) se retrouvent ainsi à la fois chez les acteurs opérationnels et chez les habitants. La notion d'altérité territoriale permet donc d'insister ici non pas sur une catégorisation type (rural, urbain, etc.) mais bien sur la préoccupation des personnes interrogées de décrire ce qui différencie leur territoire des autres.

\section{LA COMPLEXIFICATION DU RAP- PORT À L'ESPACE ET LES CAPACITÉS REQUISES POUR LA MAÎTRISER}

\section{A. Valoriser la différenciation territoriale pour maîtriser les agencements}

Les quatre dimensions de l'altérité territoriale identifiées ci-dessus traduisent l'importance des interactions, des trajectoires et stratégies territoriales des habitants. En définitive, ces agencements très individualisés que l'on retrouve dans une série de dessins qui ont été réalisés par les enquêtés démontrent que les rapports aux espaces dépendent du rapport à la famille, à l'emploi, des mobilités et de la recherche/consommation de nature et de loisirs. Certes, chaque territoire se voit attribuer par les individus des attributs distincts selon les pratiques et expériences déployées (comme l'illustre la Figure 2); mais il semble essentiel de considérer certains ressorts identiques quant au besoin de qualifier le rapport aux profils spatiaux et surtout les interactions entre eux :

- Le caractère très multi-territorial des modes d'habiter, à l'échelle d'une vie (mobilité résidentielle), des pratiques annuelles (lieux ressources familiaux, chez des amis) jusqu'à l'échelle journalière, combiné à un sentiment d'appartenances multiples et emboitées (on se positionne par rapport au village, à la ville d'à côté, à la métropole qu'on a quittée). (Sencébé, 2011).

- L'importance de lieux d'intimité sur lesquels les personnes s'appuient pour ensuite décrire le monde (par exemple une estive ou un lieu de vacances privilégié, une maison dont on n'a jamais bougé). Espace ressource qui semblerait organiser la hiérarchisation affective des territoires, ces lieux d'intimité ou d'engagement, 
de relations sociales correspondent au lieu où l'on vit, quel qu'il soit. C'est aussi cela qui légitime le «bien vivre» où l'on est installé(e).

- Des attentes sur la qualité de vie partagées quel que soit le territoire de résidence, souvent attribuée à la proximité - qu'elle soit physique ou sociale - comme l'illustrent ces deux citations très proches, l'une d'un habitant d'un village pyrénéen, l'autre d'une habitante de la métropole toulousaine :
«Ici, c'est paisible, une vie assez sympa, pas besoin de prendre la voiture pour aller chercher le pain à midi » (Stéphane, Seix, chef d'entreprise, 26 ans) "C'était important pour moi, dans cet appartement, d'avoir une vue sur quelque chose de vert quand je suis à la fenêtre. Et je suis contente d'avoir tout à proximité, donc à pied, à vélo... Et de pas prendre la voiture pour aller acheter du pain, pas prendre la voiture pour faire les courses » (Elisabeth, Toulouse, graphiste, 32 ans).

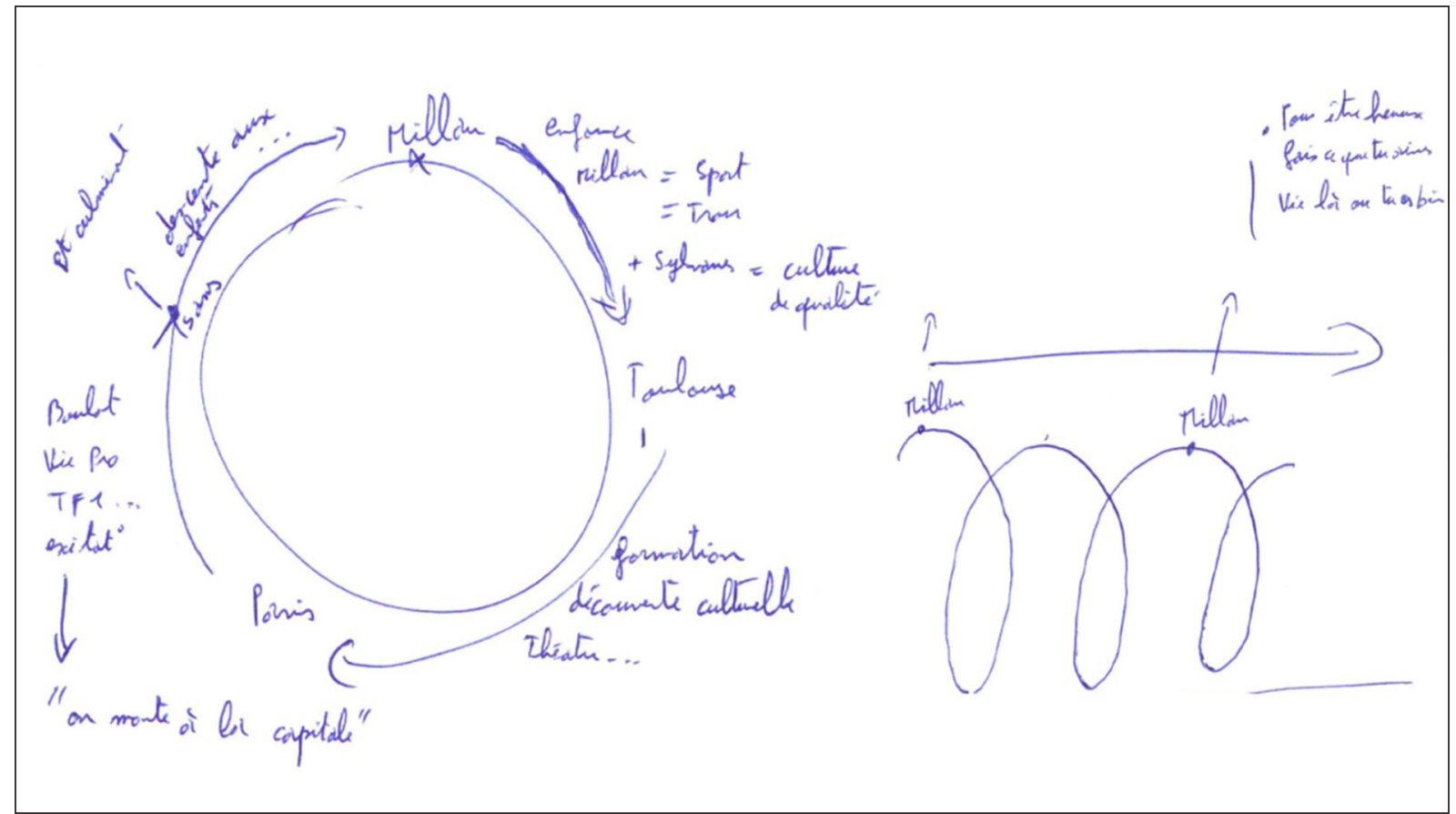

Figure 2. L'exemple de multiples agencements individuels

Enquêteur. - Donc Millau, Toulouse, Paris. SAM. - Oui. Toulouse... Formation. Découverte culturelle. On parlait bien de Millau... Je pense que je vais faire un truc... On reste dans le domaine de Millau, parce que sinon, il y a un endroit qui est important pour moi, qui est à une heure d'ici. C'est une abbaye, qui s'appelle Sylvanès, qui est un centre culturel, qui a été... Où il y a beaucoup de concerts, beaucoup de stages, de culture. Enfin, en Aveyron, tu as Conques et Sylvanès, qui sont deux gros lieux culturels. En passe de devenir, d'ailleurs, centre culturel européen. Un gros gros truc. Et moi, l'aspect culturel qui m'a manqué à Millau, j'ai tout trouvé là-bas. Voilà. C'est le complément de Millau, même si c'est à une heure. Et les gens ne connaissent pas beaucoup à Millau. Pour tout ce qui est musique... Parce que je suis très branché... Enfin, je t'en ai pas parlé, mais j'ai un domaine de ma vie qui est musique, aussi. Musique classique. Et j'ai tout découvert là-bas! Plus, on va dire, Sylvanès... Ensuite... Paris. Alors, ici... Boulot, vie pro. J'ai bossé à TF1 aussi. Je vais le mettre. C'est un peu dans l'idée de... Voilà, c'est la grande ville... Donc vie professionnelle, excitation. Je vais même te mettre la petite phrase : on monte à la capitale. Et moi, j'ai pu... Moi, ce qui m'a sauvé, c'est que j'ai fait faillite, entre guillemets. Sinon, j'y serai.

Ici, ça, c'est le point culminant. Cinq ans, d'ailleurs. Descente aux enfers! J'exagère un peu, mais c'est un peu ça. On schématise. Et retour à Millau.

Enquêteur. - Voilà.

SAM. - Sauf que si on le voit sur une spirale... Si tu la vois comme ça, ta spirale, ça fait un cercle. Mais sauf qu'entre-temps, il y a eu une très grande progression.

Enquêteur. - D'accord.

SAM. - On part bien de Millau ici... Il y a plu- 

singularités et des pratiques socio-spatiales dans l'action locale

sieurs spirales. Enfin, bon, on arrive à Millau ici. Et il y a eu... Et c'est pas le même Millau. Ce Millau-là, c'est... Millau de là ! T'as compris. Enquêteur. - Oui, c'est le départ.

SAM. - Et le Millau d'arrivée, c'est : pour être heureux, fais ce que tu aimes et vis là où tu es bien. Voilà.

Extrait de Sam, 40 ans, Millau

Dans leur très grande majorité, les discours recueillis soulignent finalement le besoin de valoriser le territoire vécu par la différenciation relative à d'autres : "Rien que ça, les enfants peuvent aller à l'école à pieds alors qu'on serait encore à Castelginest, je ne laisserais pas mes enfants aller à l'école à pieds : on entend tellement de choses ... Mais ici, même si on ne les a jamais laissés aller à pieds, ça me poserait moins de problèmes ! » (Patrice, 41 ans et directeur d'entreprise, vit à Seix depuis 11 ans). Jules (26 ans, Saint Grégoire, garagiste) nous dit " je me demande comment des gens peuvent vivre dans des lieux aussi reculés" pour parler des causses, alors que Patricia qui vit à Saint-Girons depuis 9 ans considère : "Le périph à Toulouse ou les couloirs du métro à Paris : c'est vraiment ce que je ne voulais pas vivre! Pour moi c'est l'anti chambre de l'enfer ... ». (34 ans, Chargée de mission dans une communauté de commune).

\section{B. Capacités habitantes et capital d'autochtonie multi-situé : quelle perspective dans le dévelop- pement territorial ?}

L'altérité territoriale implique ainsi à la fois la présence de délimitations, voire de frontières et la valorisation du fait de les avoir franchies dans un sens ou un autre. Il semble que pour cela, les pratiques décrites par les personnes interrogées s'inscrivent dans trois champs de capacité, qui ne sont pas spécifiques d'une catégorie spatiale ou d'une autre. C'est d'abord la capacité de mobilité (y compris par des moyens mineurs : autostop par exemple) qui permet d'être à la fois « d'ici » bénéficiant des aménités et offre de proximité (de relations, de services, d'emplois), mais aussi de pouvoir précisément élargir le territoire de vie, et donc de ne pas être « captif » d'une catégorie. C'est ensuite la capacité d'adaptation des individus à des changements de situation dans le quotidien : aux nouveaux profils d'emploi, ou encore à l'amélioration de la qualité alimentaire par exemple. Enfin, la capacité d'intégration dans des réseaux d'entraide, de vie collective, de ressources locales est primordiale.

Ces différentes capacités rencontrent des éléments déclinant le capital d'autochtonie (Rétière, 2003 ; Rénahy, 2010) : celui-ci permet, selon les auteurs, d'accéder à des formes de reconnaissance qui sont devenues aujourd'hui difficilement accessibles dans la sphère professionnelle, voire qui présentent une alternative à des compétences plus académiques ou socio-culturelles (Mazaud, 2010).

Le capital d'autochtonie se fonde sur une reconnaissance locale alliée à la connaissance locale : famille, amis, figures locales qui permettent aux habitant de se doter de ressources relationnelles, de s'inscrire dans des réseaux de sociabilité locaux pour accéder à l'emploi, pour rencontrer un conjoint, pour s'ancrer dans un territoire où on élit domicile. Mais au-delà, on voit que plus cette capacité est multi-située, mieux les habitants expriment une satisfaction à être où ils vivent (voire une satisfaction de ce qu'ils vivent). Au risque de créer une torsion avec le terme initial qui insiste sur le local au singulier, nos entretiens nous suggèrent la force d'un " capital d'autochtonie multi-situé », intégrant de ce fait la diversité des territoires investis d'une part, mais aussi le fait que fréquenter et réseauter dans plusieurs territoires contribue à alimenter les capacités citées précédemment - mobilité, adaptation, intégration. Il s'agit d'une capacité différente des individus à la maîtrise de l'altérité territoriale.

Comment intégrer ces différentes notions dans une perspective de développement territorial et d'action locale? La complexification des modes de vie et des problèmes posés (transition écologique, précarité de l'emploi, nouvelles configurations familiales, etc.) nécessiteraient des méthodes de développement territorial très singularisées, offrant des dispositifs adaptables et surtout valorisant la diversité des partenariats et objectifs de développement (pourquoi toujours vouloir attirer de la population et des activités ?). Dans une perspective de développement territorial intégrant les singularités situées de chaque territoire, les catégories spatiales n'expliquent ni les problèmes sociaux, ni les problèmes de vivre ensemble, ni les problèmes démocratiques. Cela interroge particulièrement les dispositifs d'action publique et la formulation des enjeux qui y sont associés : comment répondre aux objectifs de bien vivre dans les territoires si ceux-ci 
sont appréhendés selon des critères homogénéisants ou caricaturaux ? C'est par exemple ce que suggère le zonage de la précarité récemment utilisé : le carroyage de la pauvreté ayant permis de repérer la très forte concentration de pauvreté pour établir les nouveaux périmètres de la politique de la ville ne peut amener à ignorer la pauvreté moins prédominante mais bel et bien problématique ailleurs : " Si l'on veut mettre en place des politiques qui répondent aux besoins, il y a urgence à mieux comprendre le territoire et faire la part des choses. Surtout, les réponses à apporter dans ce domaine aux jeunes, aux familles (monoparentales en particulier) ou aux personnes âgées diffèrent bien davantage par leurs caractéristiques démographiques que par leur lieu de vie.» (Centre d'observation de la société, 2018). C'est cette complexification de la lecture territoriale que souligne cet article. En cela, nous rejoignons E. Charmes : «On ne peut pas essentialiser le spatial et en faire le creuset du rapport au monde ».

Dans cette perspective, la compréhension fine de l'altérité territoriale et des différentes capacités identifiées pour la maîtriser semblent essentielles à considérer en faveur d'un développement territorial intégrant la singularité des territoires et leurs interactions. C'est pourquoi le capital d'autochtonie multi-situé nous semble important à considérer si l'on suit les logiques d'interterritorialité qui semblent opportunes à privilégier pour intégrer les différents défis en cours (Vanier, 2008) : habitat, emploi, mobilité, loisirs, gestions des ressources naturelles, etc. En ce sens, trois leviers s'imposent pour spécifier les trajectoires territoriales selon leur épaisseur socio-spatiale, mais aussi les interactions que construisent les pratiques socio-spatiales :

- L'ingénierie territoriale qui est de plus en plus déterminante dans la capacité des territoires à réagir aux problématiques posées ;

- La formation des élus quant aux problématiques territoriales et sociétales, ce qui permettrait de mieux spécifier les objectifs et le sens du développement ;

- L'intégration des individus et de leurs parcours, de leurs représentations et de leurs besoins dans l'élaboration de l'action publique - ce qui en fait un enjeu clairement démocratique.

Cela étaye plusieurs recherches ayant montré que la structuration de l'action territoriale était liée à la capacité de systèmes d'acteurs locaux à valoriser des ressources spécifiques (Pecqueur, 2014), à organiser et mobiliser une ingénierie de développement (Joyal et al., 2011) et à s'approprier les effets leviers de politiques nationales et européennes (Taulelle, 2012).

\section{CONCLUSION}

À travers 1'analyse de discours produits dans des espaces très contrastés, les objectifs du programme du Labex SMS Interactions permettent de contribuer au débat sur la diversité territoriale. Contrairement à des considérations académiques très relayées à l'échelle médiatique, le « tous urbains, tout urbain » ne semble pas être intégré dans certaines réalités locales et dans les modes $d$ 'habiter. Les entretiens avec des habitants et avec des acteurs opérationnels traduisent une complexité des réalités territoriales et des interactions, qui ne semblent pas intégrées dans les discours dominants sur les territoires et leurs contrastes. Cela confirme ce que d'autres travaux basés sur des approches qualitatives indiquent, c'est à dire la nécessité de sortir de l'idée d'un archétype unique de pratiques urbaines ou périurbaines. Cailly et Dodier (2007) insistent sur la diversité des modes d'habiter dans les couronnes périurbaines, Ortar (2008) souligne les différenciations de genre et Giroud et al. (2011) ont mis en lumière des formes de périurbanisation particulières au périurbain des villes moyennes. Les démarches qualitatives ont aussi permis de confirmer la pérennité de la grille de lecture ville-campagne (Alduy, 2006) ou urbain-rural (Morel-Brochet, 2007). Plus récemment, des travaux sur les modes d'habiter nous montrent également que les campagnes des villes tout comme les villes des campagnes sont majoritairement représentées et appropriées comme rurales par leurs habitants (Poulot, 2015 ; Coquard, 2016, Barthe et al., 2017). Cette production de connaissances sur la diversité des modes d'habiter au sein de catégories spatiales permet de cerner ce que nous appelons «l'altérité territoriale », à savoir une certaine conscience des habitants et des acteurs de la différenciation territoriale, de leur positionnement spatial et de leur aisance à se jouer des catégories d'espace, en les agençant tant quotidiennement qu'occasionnellement.

En définitive, les catégories spatiales officielles, celles de l'Insee par exemple, semblent assigner les territoires à des modèles de développement 

singularités et des pratiques socio-spatiales dans l'action locale

selon quelques caractéristiques et leur trajectoire précédente (path dependency), alors même que des ressources nouvellement activées peuvent être à l'origine de pistes de développement inédites. Comment le développement territorial peut-il se saisir d'un côté des catégories spatiales relativement arrêtées et de la complexité identifiée en observant les modes d'habiter? Mettre en avant ces modes d'habiter et les capacités mobilisées, c'est, nous semble-t-il, une condition pour relever les nouveaux défis de l'action locale - par exemple les inégalités à venir sur le réchauffement climatique ou la capacité d'adaptation aux nouveaux cadres du marché de l'emploi avec en particulier le déploiement du télétravail ou la saisonnalité de l'emploi. Prendre en compte le capital d'autochtonie, c'est aussi réfléchir à la manière dont peut se construire, sur des méthodes plus contributives, des projets de territoires qui ne se fondent donc plus sur des catégories spatiales mais bien sur des problématiques sociales, qu'il convient de mettre en débat (place de la nature, solidarité locale, type de mobilités, etc.).

Aujourd'hui, habiter des territoires relève d'une série de capacités, qui valorisent et traduisent la maîtrise de ce que nous avons donc qualifié d'altérité territoriale. Aussi l'intégration des catégorisations habitantes dans la réflexion permet de remettre en question les injonctions des cadres de l'action publique territoriale (intercommunalités XXL à marche forcée dans les espaces ruraux par exemple) et de mieux les faire correspondre à leur appropriation locale effective.

En termes d'actions, les défis pour les élus et les techniciens sont d'autant plus importants que cela renvoie à la difficile actualisation de leurs connaissances de la complexité de la société dans laquelle les pratiques s'individualisent et dans laquelle il y a une perte des liens sociaux traditionnels. C'est particulièrement vrai en Occitanie, dont le solde migratoire est largement positif, impliquant un fort renouvellement de population aux profils très contrastés, y compris dans des territoires considérés comme moins dynamiques. Plus largement, les dernières actualités sanitaires - donc sociales et économiques - ont montré à quel point la diversité territoriale était vivace : intégrer les modes d'habiter et les réalités socio-spatiales semble incontournable pour des réponses nécessairement différenciées de l'action publique.

\section{REMERCIEMENTS}

Ce travail est issu du projet «Interactions, les dynamiques territoriales entre sphères urbaine et rurale " financé par le Labex Structuration des Mondes Sociaux de l'Université de Toulouse. Nous remercions les autres chercheurs ayant participé à la réalisation des enquêtes et aux discussions : L. Barthe, J. Bessière, F. Cavaille, I. Duvernoy, F. Escaffre, C. Eychenne, C. Jebeili, F. Laumière, P. Panegos, F. Taulelle.

\section{BIBLIOGRAPHIE}

Alduy, J.-P. (2006). Identité, catégorisation socio-spatiale et mobilité : être urbain et se penser rural ? Travaux de l'Institut de Géographie de Reims, 115-118, 45-58.

Arlaud, S., Jean, Y. \& Royoux, D. (coord.) (2005). Rural-Urbain. Nouveaux liens, nouvelles frontières. Presses universitaires de Rennes.

Ascher, F. (1995). Métapolis, ou l'avenir des villes, Paris, Odile Jacob.

Barthe, L., Gambino, M., Laumière, F. \& Sibertin-Blanc, M. (2017). L'inégale appropriation des catégories spatiales urbain/rural en Midi-Pyrénées. In $\mathrm{M}$. Berger, J.-L. Chaléard (coord.), Villes et campagnes en relation. Regards croisés Nords-Suds, Paris, Karthala.

Behar, D., Dang Vu, H. \& Delpirou, A. (2018). France périphérique, le succès d'une illusion. Alternatives économiques.

Bouba-Olga, O., Chauchefoin, P., Chiron, H., Ferru, M., Guimond, B. \& Nadau, E. (2017). Dynamiques territoriales. Éloge de la diversité. Éditions Atlantique, $100 \mathrm{p}$.

Cailly, L. \& Pourtau, B. (2018). Faire métropole : une analyse par les représentations et les pratiques de mobilité périurbaines des habitants de l'aire urbaine de Tours (France). Géocarrefour [En ligne], https:// journals.openedition.org/geocarrefour/10394

Cailly, L. \& Dodier, R. (2007). La diversité des modes d'habiter des espaces périurbains dans les villes intermédiaires : différenciations sociales, démographiques et de genre. Norois, 205, 67-80.

Campagne, P. \& Pecqueur B. (2014). Le développement territorial. Une réponse émergente à la mondialisation. Éditions Charles Léopold Mayer.

Cattan, N. (2017). Introduction : Une politique d'aménagement durable des territoires ne peut ignorer l'urbain dispersé. In A. Bres, F. Beaucire, B. Mariolle (dir), Territoire frugal, La France des campagnes à l'heure des métropoles, Lausanne, MétisPresses.

Centre d'observation de la société (2018). Les troisquarts des pauvres vivent hors des quartiers prioritaires. http://www.observationsociete.fr 
Charmes, E. (2011). La ville émiettée. Essai sur la clubbisation de la vie urbaine, PUF.

Charmes, E. (2017). La revanche des villages. La vie des idées.

Coquard, B. (2016). Paris ? Jamais de la vie. Goûts et dégoûts territoriaux chez les jeunes ruraux de classes populaire. Savoir/Agir, 37, 39-45.

Di Méo, G. (1991). L’Homme, la société, l'espace. Paris, Anthropos/Economica, 319p.

Di Méo, G. (1996). Les territoires du quotidien. L'harmattan, 207p.

Joyal, A., Lardon, S. \& Dayan, L. (2011). L’ingénierie de territoire à l'épreuve du développement durable. L'Harmattan.

EUROSTAT (2016). Urban europe, statistics on cities, towns and suburbs. Statistical book.

Estebe, P. (2015). L'égalité des territoires, une passion française. PUF, coll. La ville en débat, 88p.

Giroud, M., Mainet, H. \& Édouard, J.-C. (2011). Les mobilités spatiales dans les villes intermédiaires. Territoires, pratiques, régulations. Clermont-Ferrand, Presses universitaires Blaise Pascal.

Hervieu, B. \& Viard, J. (2001). L'archipel paysan. La fin de la république agricole. La Tour d'Aigues, Éditions de l'Aube, 125p.

Hilal, M., Barczak, A., Tourneux F.-P., Schaeffer, Y., Houdart, M. \& Cremer-Schulte, D. (2012). Typologie des campagnes françaises et des espaces à enjeux spécifiques. Synthèse, DATAR (2012). 80p.

INSEE (2011). Le nouveau zonage en aires urbaines de 2010. Insee Première, $n^{\circ} 1374,4 p$.

INSEE (2012). Le nouveau zonage en bassins de vie de 2012. Trois quarts des bassins de vie sont ruraux. Insee Première, $\mathrm{n}^{\circ} 1425,4 \mathrm{p}$.

INSEE (2014). Une approche de la qualité de vie dans les territoires. Insee Première, $\mathrm{n}^{\circ} 1519,4 \mathrm{p}$.

Jebeili, C. (2019). Cadre théorique et pratique de la différenciation territoriale en droit. Séminaire Différenciations territoriales et action collective, LISST.

Levy, J. (2013). Réinventer la France. Trente cartes pour une nouvelle géographie, Fayard.

Lussault, M. (2018). Tous urbains ! dans AOC média, rubrique Opinion, le 7 février.

Mazaud, C. (2010). Le rôle du capital d'autochtonie dans la transmission d'entreprises artisanales en zone rurale. Regards sociologiques, 40, 45-57.

Morel-Brochet, A. (2006). Ville et campagne à l'épreuve des modes d'habiter. Approche biographique des logiques habitantes. Thèse de Doctorat, Université Paris 1. En ligne : https://tel.archives-ouvertes.fr/ tel-00264308/file/TheseAMorelBrochet2006.pdf

Morel-Brochet, A. (2007). À la recherche des spécificités du mode d'habiter périurbain dans les représentations et les sensibilités habitantes. Norois, 2007, 23-35.

Offner, J.M. (2019). « Gilets jaunes » : une politique du quotidien reste à inventer, seule à même de répondre au mal-être exprimé. Le Monde, 15 janvier.

Ortar, N. (2008). Entre ville et campagne, le difficile équilibre des périurbaines lointaines. Métropoles, 18.09.2008.

Poulot, M. (2015). Être ou ne pas être rural... quand le rural se décline en ville comme à la campagne. Pour, 228, 69-76.

Renahy, N. (2010). Classes populaires et capital d'autochtonie. Regards sociologiques, 40, 9-26.

Retiere, J.-N. (2003). Autour de l'autochtonie. Réflexions sur la notion de capital social populaire. Politix, 63(16), 121-143.

Sencebe, Y. (2011). Multi(ples) appartenances en milieu rural. Informations sociales, 2011/2(164), 36-42.

Talandier, M. \& Pecqueur, B. (2018). Renouveler la géographie économique. France. Economica.

Taulelle, F. (coord.) (2012). Le délaissement du territoire : quelles adaptations des services publics dans les territoires ruraux. Sciences de la société, 86.

Vanier, M. (2008). Le pouvoir des territoires. Essai sur l'interterritorialité. Anthropos-Economica.

Coordonnées des auteurs :

Mélanie GAMBINO

Maître de conférences en géographie, LISST-DR

Université de Toulouse Jean Jaurès, France melanie.gambino@univ-tlse2.fr

Mariette SIBERTIN-BLANC

Maître de conférences en géographie et aménagement, LISST-CIEU

Université de Toulouse Jean Jaurès, France mariette.sibertin-blanc@univ-tlse2.fr 